\title{
Banāt al-barārī de Mahā Ḥasan : corps et fantastique
}

Mahā Hasan's Banāt al-barārī : body and fantastic

$$
\text { بنات البراري لمها حسن : الجسد والفنتستيك }
$$

\section{Martina Censi}

\section{(2) OpenEdition}

\section{Journals}

Édition électronique

URL : http://journals.openedition.org/beo/1350

DOI : 10.4000/beo.1350

ISSN : 2077-4079

\section{Éditeur}

Presses de l'Institut français du Proche-Orient

\section{Édition imprimée}

Date de publication : 30 avril 2014

Pagination : 171-186

ISBN : 978-2-35159-403-2

ISSN : 0253-1623

\section{Référence électronique}

Martina Censi, «Banāt al-barārī de Mahā Hasan : corps et fantastique », Bulletin d'études orientales [En ligne], LXII | 2014, mis en ligne le 04 juin 2014, consulté le 01 mai 2019. URL : http:// journals.openedition.org/beo/1350 ; DOI : 10.4000/beo.1350 


\section{Banāt al-barārī de Mahā Ḥasan : corps et fantastique}

Martina CENSI

Université de Catane, CERMOM de (INALCO)

\section{Le corps dans le roman des écrivaines syriennes contemporaines}

Dans ma thèse de doctorat, intitulée Les représentations du corps dans le roman des écrivaines syriennes contemporaines, j'ai envisagé les différentes représentations du corps masculin et féminin dans une sélection de romans en langue arabe écrits entre 2004 et 2011 par des écrivaines syriennes contemporaines ${ }^{1}$. L'analyse de ces romans m'a permis de mettre en évidence le fait que l'élément corporel en affectait à la fois le sujet et le langage. Je me suis alors posé la question suivante : pourquoi ces écrivaines réservent-elles un espace spécifique à la dimension corporelle ? Et quelle est sa fonction?

L'hypothèse qui a été avancée, et que j'ai eu l'occasion de vérifier au cours de ma recherche, est qu'aujourd'hui, à travers la littérature, et notamment le roman, l'intérêt des écrivaines syriennes s'éloigne de la question de l'émancipation féminine pour se porter sur un phénomène plus général impliquant aussi bien l'homme que la femme. Le regard n'est pas dirigé vers le corps en lui-même, mais vers le corps en tant que représentation fondamentale de l'individu. Le corps est le lieu de négociation entre instances individuelle et collective et il joue un rôle central comme métaphore de l'individu en tant que « sujet désirant », par opposition au concept d' " assujetti », c'est-à-dire l'être humain qui, sous la double action de la répression politico-religieuse et des pressions de la communauté, perd son moi et ainsi son statut d'individu.

Dans les romans de ces écrivaines apparait un renouvellement de l'intérêt pour la dimension privée, laquelle acquiert un sens différent par rapport à la façon dont elle était traitée dans la fiction des générations précédentes. Si on envisage par exemple la

1 Thèse soutenue à l'Université Ca' Foscari de Venise en cotutelle avec l'Institut National des Langues et Civilisations Orientales de Paris, sous la direction de $\mathrm{M}^{\text {me }}$ Antonella Ghersetti et M. Sobhi Boustani. Mon corpus comprenait notamment les romans suivants : Kursī de Dīma Wannous, Hurrās al-hawāà de Rozā Yāsīn Hạan, Banāt al-barārī de Mahā Ḥasan, Rā̉iḥat al-qurfa de Samar Yazbik et Imrảa min hāêā al-'așr de Hayfā' Bīṭār. 
production romanesque de pionnières comme Widād Sakākinn $\overline{1}^{2}$ et Colette al-Huūrī ${ }^{3}$, on y trouve déjà des descriptions de scènes érotiques qui restent toutefois timides et allusives. Dans les années soixante-dix, on trouve le cas spécifique d'al-Riwāya al-mal'ūna (Le roman maudit), écrit en 1967 par l'écrivaine syrienne Amal Ǧarrāḥ, dans lequel l'auteure raconte le désir incestueux d'une adolescente pour son père. Mais, même ici, la dimension corporelle n'est pas décrite de façon détaillée.

$\mathrm{Au}$ contraire, si l'on considère les romans plus récents qui ont fait l'objet de ma recherche doctorale, on remarque que le corps est abordé de façon directe et décrit dans ses moindres détails. C'est notamment le cas dans le roman Kursī de Dīma Wannūs, qui y décrit un processus de fragmentation du corps du protagoniste et le décompose en une série de fonctions physiologiques, avec pour objectif de représenter les effets du pouvoir despotique sur l'individu. C'est également le cas, bien que de façon différente, dans Hurrās al-hawā' de Rūzā Yāsīn Hasan, où cette dernière analyse le corps à travers plusieurs dimensions, en tant que lieu métaphorique de l'expérience de la prison et de la répression politique en Syrie. Samar Yazbik, dans Rảihat al-qirfa, aborde quant à elle le sujet de l'homosexualité féminine, qui devient le seul espace possible dans lequel la femme peut s'affirmer librement au cœur d'une société qui empêche la création de liaisons libres entre homme et femme. La dimension de la sexualité est choisie par l'auteure pour représenter les relations hétérosexuelles, toujours caractérisées par la violence, ainsi que les relations homosexuelles dans lesquelles l'érotisme réussi à se développer et à s'exprimer.

Ces écrivaines ne se contentent pas de représenter le corps dans sa dimension sexuelle, mais en approfondissent aussi les autres dimensions comme celles de la maladie, de la vieillesse, de la grossesse, de la torture et des fonctions physiologiques. En outre, elles ne se focalisent pas seulement sur le corps de la femme mais s'intéressent aussi au corps masculin. Le corps n'est ainsi plus utilisé seulement comme moyen de représentation de la condition de la femme, mais plutôt pour analyser la relation entre l'individu et la collectivité.

Pour rendre compte du changement d'approche des romancières syriennes par rapport au corps, j'ai choisi le cas de Banāt al-barārī (Les filles des terres sauvages, 2013) de Mahā Hasan ${ }^{4}$, car il s'agit d'un exemple particulièrement représentatif de ce phénomène. Dans ce roman, l'auteure a recours au registre fantastique pour représenter la position du corps entre communauté et nature. Le regard n'est pas dirigé vers le corps en lui-même, mais vers le corps en tant que représentation de l'individu et lieu de la négociation entre instances individuelle et collective. Dans ce lieu métaphorique, le « sujet désirant » cherche à s'affirmer en refusant son rôle d'« assujetti ».

2 Pour une étude approfondie de l'oeuvre de cette auteure, voir BITAR OTTOSSON 2005.

3 Voir ZEYDAN 1995, p. 104-111.

4 Née à Alep, Mahā Hasan est écrivaine et journaliste d'origine kurde. En 2000, il lui a été interdit de publier ses œuvres en Syrie. En 2004, elle s'est installée à Paris où elle vit et travaille actuellement. Elle se consacre principalement au roman. Ses œuvres se caractérisent par l'attention portée à des sujets historico-politiques qui interrogent la société syrienne. 


\section{Entre réalisme et fantastique}

Dans Banāt al-barārī, Mahā Hasan raconte l'histoire de deux femmes partageant le même destin. L'amour les conduit à une fin tragique : toutes deux sont victimes de crimes d'honneur. Pour aborder le sujet du crime d'honneur, choisi comme métaphore de l'oppression exercée par la tradition sur l'individu, l'auteure a recours à une interaction entre langage fantastique et langage réaliste, dans un roman qui se présente comme une véritable œuvre de critique sociale. Le corps, présenté à la fois dans la dimension de la souffrance et du plaisir, est le lieu dans lequel se déroule la lutte entre les instances individuelle et sociale. Dans le roman, le personnage n'arrive pas à créer un espace pour s'affirmer en tant qu'individu, car il est dominé par le contrôle imposé par la communauté, symbolisé par le crime d'honneur.

Dans Banāt al-barārī, le crime d'honneur perd sa connotation liée au gender ${ }^{5}$ pour devenir la métaphore de l'oppression que la tradition exerce sur la société dans son ensemble. Les personnages masculins du roman sont eux-mêmes victimes du phénomène. Ainsi, l'amant ne peut éviter l'assassinat de sa femme, et il en va de même pour le père, qui se voit contraint de tuer sa fille pour sauvegarder l'honneur de la famille.

L'auteure s'appuie sur le registre fantastique, qui se manifeste également dans l'organisation de l'œuvre. Chaque chapitre est identifié par le nom d'une couleur possédant un signifié spécifique, dans une sorte de symbolique alternative. La narration commence avec un événement à caractère réaliste - l'assassinat de Sulțāna, égorgée car tombée enceinte en dehors du mariage - avant d'acquérir un caractère relevant du fantastique, autrement dit non explicable rationnellement. Le cadavre de la jeune fille, suspendu à la porte de la maison, subit un miracle : la tête se réunit au corps, et le cadavre prend entre ses bras le nouveau-né. La deuxième manifestation étrange est l'apparition de la couleur rouge - celle du sang de la femme égorgée - qui envahit tout le village en effaçant les autres couleurs. L'esprit de Sulțāna ne retrouve la paix qu'après le retour de son amant, Ibrāhīm, qui lui promet de s'occuper de leur fille.

L'intrigue est partagée entre deux ordres d'événements : ceux qui se rattachent au réel et ceux qui se rattachent au fantastique. Ces deux niveaux ne restent pas toujours opposés l'un à l'autre au cours de la narration. Et c'est exactement dans cette ambiguïté que se manifeste le caractère fantastique du roman ${ }^{6}$. Dans son analyse de Fuqaha al-zalām de l'écrivain syrien Salīm Barakāt, Boutrous Hallaq (2001) soutient que le fantastique se détache de sa définition de genre littéraire "affreux », en devenant un vrai moyen de connaissance. En établissant un dialogue avec le réel, le fantastique suscite l'istiğrāb

$5 \quad$ Le terme gender traduit toutes les constructions symboliques et socio-historiques qui agissent sur le donné biologique du corps et nous amènent à percevoir les rôles des deux sexes comme quelque chose de naturel et immuable. Le gender est aussi l'outil qui nous permet d'analyser la construction sociale et discursive qui se rattache au corps. Voir SCOTT 1986.

6 Voir notamment Todorov 1970. Relèvent du registre fantastique les événements qui ne peuvent recevoir d'explication rationnelle et qui produisent un effet particulier sur le personnage et le lecteur : hésitation, peur, horreur ou simplement curiosité. Ce genre entretient le suspense, car ni le personnage ni le lecteur n'arrivent à distinguer la réalité de l'imaginaire. 
(étonnement) et le tasäul (interrogation) 7 . La recherche de l'explication du phénomène exceptionnel implique aussi bien le personnage que le lecteur. Dans le cas de Banāt al-barārì, ce dernier, dépaysé à cause de l'infraction au pacte narratif, se retrouve suspendu entre deux genres littéraires : celui du roman de critique sociale et celui du roman fantastique.

Le fantastique se manifeste également dans le roman à travers le conte de fées, utilisé pour expliquer un phénomène en contradiction avec les lois de la nature. L'écrivaine reprend le genre du conte lié à la tradition orale et en retravaille le sens. Le conte, normalement utilisé pour mettre en garde les filles et défendre leur virginité ${ }^{8}$, devient ici un moyen de dénoncer le crime d'honneur. En outre, à travers ce procédé, l'auteure discute la double distinction entre culture savante et culture populaire, culture écrite et culture orale, qui a été historiquement exploitée pour marginaliser la femme au niveau artistique et littéraire (BRAIDOTTI 2002, p. 60). Le passage suivant est particulièrement représentatif de ce procédé (p. $\left.86-87^{9}\right)$ :

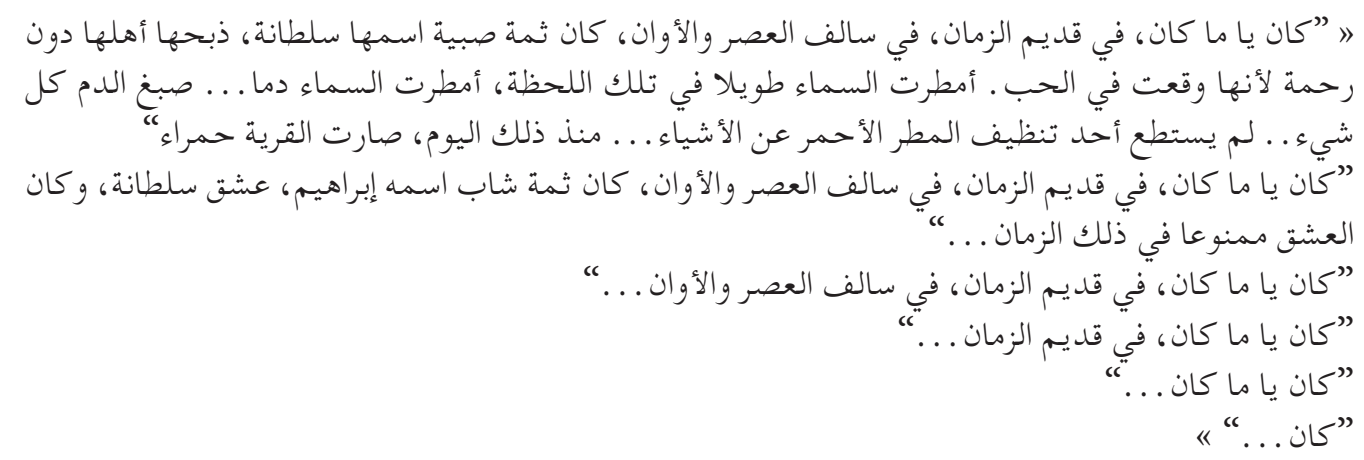

«"Il était une fois, il y a bien longtemps, une jeune fille appelée Sulțāna, laquelle fut égorgée sans pitié par sa famille, parce qu'elle était tombée amoureuse. A partir de cet instant, il plut longtemps, il plut du sang... Le sang teignit toute chose... Personne ne put nettoyer la pluie rouge... Et depuis ce jour-là, le village devint rouge."

"Il était une fois, il y a bien longtemps, un jeune homme appelé Ibrāhīm ; il aima Sulțāna, mais en ce temps-là, l'amour était interdit..."

"Il était une fois, il y a bien longtemps..."

"Il était une fois, il y a longtemps..."

"Il était une fois..."

"Il était..." ${ }^{10} »$

Le «il était une fois » s'insère comme une histoire dans l'histoire, pour donner une explication à l'invasion de la couleur rouge. Le fantastique ne se manifeste pas seulement au premier niveau narratif - celui de l'histoire des deux amants - mais également à l'intérieur

$7 \quad$ Boutros Hallaq se réfère à la définition de Todorov (1970).

8 Je m'appuie ici sur un entretien que m'a accordé l'auteure à Paris, le 12 mai 2012.

9 Dans la suite de l'article, les références ne mentionnant qu'un numéro de page(s) renvoient à l'édition du roman figurant dans la bibliographie.

10 Toutes les traductions ont été réalisées par mes soins. J’adresse un remerciement spécial à Éric Gautier pour sa relecture et ses suggestions. 
du deuxième niveau, c'est-à-dire celui des contes que les gens du village se racontent. Le « il était une fois » marque la frontière entre mondes réel et fantastique car, comme le suggère Jolles (2003, p. 434) :

«Le conte [de fées] est en opposition avec tout ce que l'on a l'habitude d'observer dans le monde comme événement réel, précisément puisque le monde du conte est séparé du monde de la réalité de façon plus radicale que toutes les autres formes. »

Toutefois, la désintégration linguistique que la formule « il était une fois » subit dans ce passage du roman représente l'amincissement de la frontière séparant le domaine du réel de celui du conte. Ce phénomène est engendré par le fait que, dans le roman, ces deux composantes ne sont pas nettement séparées, mais entraînées dans un rapport dialectique qui, précisément, détermine le caractère fantastique de l'œuvre.

La présence du conte n'est pas seulement un choix esthétique, mais elle joue un rôle plus complexe. À ce sujet, on peut se référer à la pensée de Calvino (2002, p. 121), qui soutient que le conte de fées est gouverné par ce qu'il appelle la "morale ingénue », c'est-à-dire la morale qui refuse l'injustice en cherchant à la compenser. Par conséquent, la structure du conte tourne autour de la réparation de l'injustice, selon la logique de cette morale ingénue qui, en s'appuyant sur un jugement émotif absolu, partage le monde entre bons et méchants (JOLLES 2003, p. 430). À ce même propos, Jolles (2003, p. 431) affirme que les manifestations de l'injustice qui caractérisent le monde réel «apparaissent dans le conte seulement pour être éliminées de façon graduelle et définitive et être dissoutes selon les lois de la morale ingénue. »

Si on analyse Banāt al-barārī à la lumière de cette définition, l'injustice se manifeste alors dans l'assassinat de Sulțāna, qui se venge sur le village à travers la malédiction de la couleur rouge. La mission de faire revenir les couleurs est assignée à sa fille, désignée au début de l'œuvre par le prénom «*** » (ainsi noté dans le roman), élevée par son père dans les terres sauvages, loin de la civilisation. À l'âge de quinze ans, la jeune fille a enfin le droit d'ouvrir une malle - un autre élément typique du conte de fées - contenant toutes les couleurs que Tamīma, la magicienne du village, lui a laissées en héritage. Elle commence donc à peindre les terres sauvages, puis le village. Durant sa mission, elle tombe amoureuse d'un jeune homme, Hamza, et, comme sa mère, fait l'amour avec lui en dehors du mariage. Même si Ibrāhīm, son père, a vécu la même histoire, il n'arrive pas à s'opposer à la force du code d'honneur et égorge à son tour sa fille.

Bien que la structure suive au début le modèle du conte - selon le schéma injusticeréparation de l'injustice - c'est finalement la tragédie qui l'emporte. Le fait que la fille subisse la même injustice que sa mère représente l'infraction au pacte narratif et l'échec du modèle du conte. En revanche, c'est grâce à cette dialectique entre réel et fantastique que le message de critique sociale du roman s'impose : le réel parait encore plus immoral et cruel s'il frôle la possibilité de la victoire de la "morale ingénue », supposée effacer les injustices. Dans certains passages du roman les deux registres s'entremêlent, en générant 
une sorte de court-circuit, comme dans le cas des contes que les gens racontent pour expliquer la mort de Sulțāna (p. 87) :
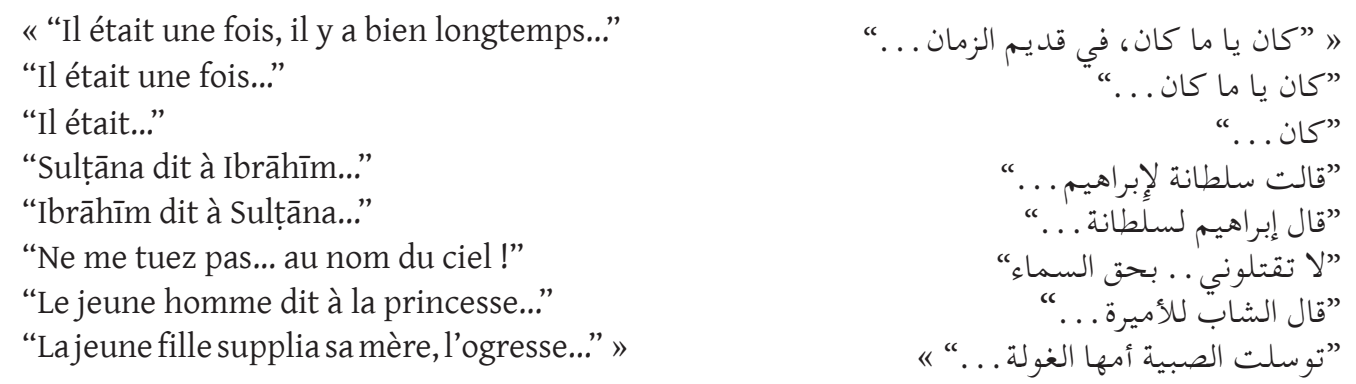

La logique du réel se manifeste notamment dans le déchirement intérieur des personnages. Leur psychologie n'est pas souvent approfondie, mais dès qu'ils se trouvent absorbés par le registre du réel, l'auteure se penche davantage sur leur univers intérieur. Et c'est à travers le changement dans le personnage d'Ibrāhīm que l'écrivaine met en place l'infraction au pacte narratif, et par conséquent la victoire du réel et de sa morale injuste. Pendant que sa fille est en train de peindre le village avec les couleurs perdues, Ibrāhīm revient aux terres sauvages. Un jour cependant, il décide de lui rendre visite au village et entend, sur le chemin, des gens parler d'une prostituée vivant avec son amant. Quand Ibrāhīm soupçonne qu'il s'agit de sa fille, et qu'il apprend que celle-ci a eu des rapports sexuels en dehors du mariage, il décide de l'égorger. La tradition remporte donc sa victoire sur l'individu, incapable d'affirmer sa propre éthique, car demeurant prisonnier de la morale collective, fondée sur la coutume.

En s'appuyant sur l'analyse de l'œuvre de l'écrivain syrien Mamdūḥ 'Azzām proposée par Darrāğ (2001), on remarque, dans Banāt al-barārī, la présence de deux structures narratives qui façonnent aussi bien la dimension temporelle que l'univers des personnages. L'une des deux suit le modèle de la hikāya, l'autre celui de la qișṣa. Selon Darrāğ (2001), la structure de la hikāya est fondée sur l'imitation et se situe dans un temps homogène et clos. On y raconte la vie en tant que sìra, un parcours linéaire dans lequel tous les événements se ressemblent. La hikīaya reproduit la cyclicité d'une société qui multiplie des histoires semblables les unes aux autres. Le personnage est un masque, emprisonné dans le monolithisme des coutumes, jamais acteur de son propre destin.

Ceci se manifeste, dans Banāt al-barārī, à travers l'histoire de la mère et de la fille partageant le même destin : toutes deux meurent égorgées pour laver l'honneur de la famille. Par contre, le personnage d'Ibrāhīm représente la dialectique entre hikāya et qișṣa : dans une partie de la narration, il incarne un modèle complexe de masculinité, alternatif à celui proposé par la société patriarcale ; mais à la fin de la narration, il se transforme en « homme animal », guidé par son instinct et dont les actions sont dictées par les traditions. La transformation du personnage est particulièrement explicite dans le passage où il commence à se douter que sa fille a perdu sa virginité (p. 132-133) : 


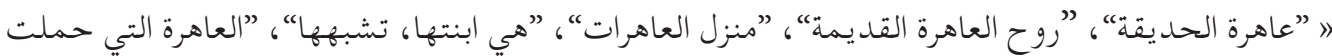

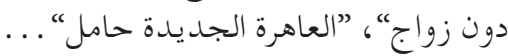

$$
\begin{aligned}
& \text { يتصاعد الدم إلى رأس إبراهيم .. يكاد يفقد عقله لـ . . يكاد يكاد يقع على الأرض، يتصور ابنته عاهرة فاتحة ساقيها } \\
& \text { كرجال القرية. }
\end{aligned}
$$

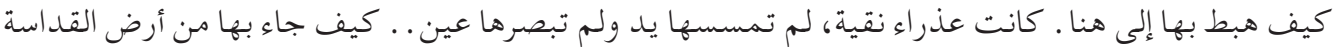

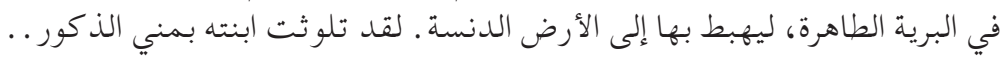

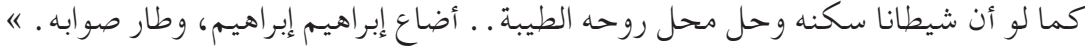

«"La prostituée du jardin", "L'esprit de l'ancienne prostituée", "La maison des prostituées", "C'est sa fille, elle lui ressemble", "La prostituée qui tomba enceinte en dehors du mariage", "La nouvelle prostituée est enceinte"...

Le sang montait à la tête d'Ibrāhīm... il était sur le point de perdre la raison... il faillit tomber par terre en imaginant sa fille, telle une prostituée, ouvrant ses jambes aux hommes du village. Comment avait-il pu tomber si bas avec elle ? Elle qui était une vierge pure que jamais main n'avait caressée, ni oeil regardée... Comment avait-il pu venir avec elle d'une terre de sainteté dans la steppe immaculée, et tomber avec elle sur une terre impure? Sa fille avait été souillée par le sperme des hommes...

C'était comme si un démon le possédait et s'était substitué à sa bonne âme... Ibrāhīm avait quitté Ibrāhīm et son bon sens s'en était allé. »

Ici le personnage perd sa personnalité et son épaisseur psychologique en faveur de la conscience collective. La fille est également identifiée au personnage de sa mère et perd sa spécificité. La structure de la hikāya remporte donc sa victoire.

Au contraire, Darrāğ (2001, p. 70) considère le personnage de la qișșa comme « libre du pouvoir de la coutume » et donc comme le sujet qui :

«[...] doit créer ses propres histoires, qui ne répètent pas ce qui s'est passé avant. Et peut-être que cette recherche, engendrant de nouveaux destins à l'intérieur des nouvelles histoires, est ce qui secoue le temps en le rendant ouvert et libéré. »

La qișșa se caractérise par son ancrage dans le temps ouvert et vivant de l'Histoire, dans lequel le personnage possède une dimension spirituelle et sa propre conscience, qui lui permettent d'échapper à la dictature des coutumes. La structure narrative de la qișsa refuse le temps despotique ainsi que les interprétations univoques, en faveur du changement. Dans le roman, cela se manifeste à travers la transgression de la linéarité temporelle grâce à l'usage de l'analepse, qui génère la multiplication des niveaux narratifs. En outre, les personnages principaux incarnent, dans certains passages, les caractéristiques du personnage de la qișșa, surtout lorsqu'ils ont la possibilité d'exprimer directement leur moi à travers le monologue intérieur, comme c'est le cas, par exemple, du père de Sultāna (p. 35-37).

L'instance narrative est caractérisée par la plurivocité. En plus du narrateur externe omniscient qui s'adresse parfois directement au lecteur pour lui expliquer les événements, l'auteure intègre plusieurs narrateurs internes qui prennent la parole pour exprimer leur état psychique. Dans ce cas, la structure de la qișs a transcende la structure cyclique de la hikāya et le personnage parvient enfin à exprimer librement son univers intérieur. 
Comme nous l'avons déjà souligné, le rôle du fantastique est central dans le roman. Le conte de fées, qui prévoit une fin heureuse, se transforme en tragédie, en montrant de façon claire son échec. Pour atteindre une fin heureuse, il faut abandonner la logique du conte. De plus, on remarque que si, dans les six premiers chapitres, les registres du réel et du fantastique s'entremêlent, ils se séparent finalement dans les deux derniers chapitres. L'avant-dernier chapitre est une sorte de narration-documentaire sur le crime d'honneur, dans lequel l'auteure se réfère au phénomène en présentant des données statistiques. $\mathrm{Au}$ contraire, dans le dernier chapitre l'auteure opte définitivement pour le langage du merveilleux ${ }^{11}$. L'état de suspense entre le réel et l'imaginaire est abandonné pour affirmer définitivement la logique du merveilleux. Dans ce dernier chapitre, les terres sauvages se transforment ainsi en une sorte de paradis des femmes dans lequel elles sont enfin libres de pratiquer l'amour et d'en jouir.

Mais quel est alors le signifié de la trace du conte dans Banāt al-barārī? On peut remarquer que celle-ci est utilisée par l'auteure pour représenter la logique traditionnelle qui vise à expliquer la réalité à travers des schémas fixes, cycliques et répétitifs, en dehors de l'Histoire. Cette logique se montre de façon particulièrement claire dans le cas d'Ibrāhīm qui, au début, incarne un modèle positif de masculinité, caractérisé par des valeurs nouvelles, tandis qu'à la fin il est réabsorbé par le système traditionnel. Quand il vit isolé avec sa fille, il parvient à acquérir des valeurs fondées sur le respect de l'autre et de sa liberté individuelle. En revanche, quand il rétablit le contact avec sa société, il se conforme à ses valeurs en revenant au rôle de l' « homme traditionnel » qui égorge sa fille pour réparer l'honneur de la famille.

Avec ce roman, par conséquent, Mahā Hasan montre que, pour parvenir à un changement dans la société arabe, il est nécessaire, avant tout, de créer un espace pour l'« individu », indépendamment de la logique communautaire. C'est seulement à travers le franchissement de la cyclicité du schéma de la hikāya et l'ouverture à la qișsa qui s'ensuit que l'individu peut prendre part à l'Histoire.

\section{Le corps entre communauté et nature}

Dans Banāt al-barārī, le corps est le lieu d'une négociation entre la pulsion collective de contrôle et la pulsion individuelle de libération. La première, liée à la tradition, vise à discipliner, soumettre et réprimer le corps dans une série de codes comportementaux. Le crime d'honneur, métaphorisé par l'image du corps mutilé, représente l'une des expressions les plus violentes du contrôle communautaire sur le corps de l'individu. La vierge ne porte pas sur elle seulement son propre honneur, mais aussi celui des ses sœurs, ainsi que la virilité des mâles de la famille (voir ZEMMOUR 2002, p. 65-76). La virginité n'est pas uniquement une condition privée, elle est aussi publique, en tant que symbole de la continuité des valeurs qui sont à la base de la communauté. Par conséquent, la vierge n'est pas considérée comme

11 Todorov (1970) affirme qu'une fois résolue l'ambiguité entre les registres réaliste et fantastique, on ne peut plus parler de genre fantastique, mais de genre merveilleux. 
un individu, mais comme un « être familial » (ZEMMOUR 2002, p. 65), et la préservation de sa virginité devient une tâche dont tous les hommes de la famille sont chargés.

À ce propos, le corps dans le roman est utilisé pour représenter, d'un côté, la pression sociale exercée sur l'individu, et de l'autre, la tentative de ce dernier de trouver un espace privé dans lequel s'affirmer à travers sa jouissance. Le roman s'ouvre avec la description du corps mutilé de Sultāna, après qu'elle ait été égorgée (p.11-12) :

$$
\begin{aligned}
& \text { " في اللحظة التي انفصل فيها الرأس عن الجسد ، سقط الرأس الثاني في الأسفل . }
\end{aligned}
$$

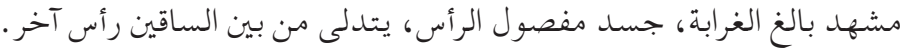

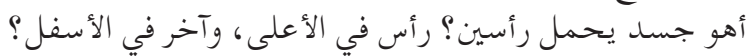

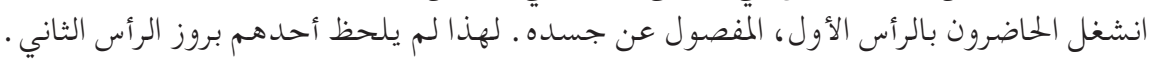

$$
\begin{aligned}
& \text { [...] } \\
& \text { الرأس الجديد ، الواصل للتو إلى الحياة،لا يصرخ، لا يعلن ميلاده، كما لو أنه عرف وأحس بأن وجوده مرفوض، }
\end{aligned}
$$

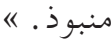

«Au moment où la tête se détacha du corps, la deuxième tête tomba.

C'était une scène extrêmement étrange : un corps sans tête et entre ses jambes une autre tête qui pend.

Le corps a-t-il deux têtes? Une tête en haut et l'autre en bas?

L'attention de l'assemblée était attirée par la première tête, détachée de son corps. C'est pourquoi personne ne remarqua l'apparition de la deuxième.

[...]

La nouvelle tête, qui venait de voir le jour, ne criait pas, n'annonçait pas sa naissance, comme si elle savait ou sentait que sa présence était refusée et bannie. »

Ici, l'écrivaine ne donne pas au lecteur les indices nécessaires pour comprendre l'image décrite, et on retrouve par conséquent l'hésitation qui, selon Todorov, caractérise le genre fantastique. Il est difficile de comprendre, à ce stade, s'il s'agit d'un roman relevant du merveilleux ou du réalisme. On remarque pourtant que la focalisation se porte sur le corps et, en particulier, sur la tête. C'est seulement dans le passage suivant que l'auteure donne d'autres éléments qui aident à interpréter la scène : une femme égorgée qui accouche. Et, peu après, la mère prend la parole en s'adressant au nouveau-né (p. 16-17) :

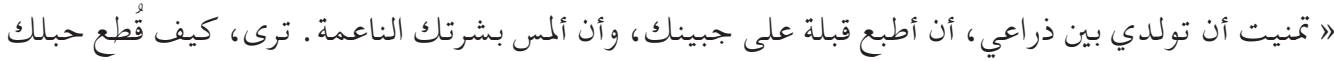

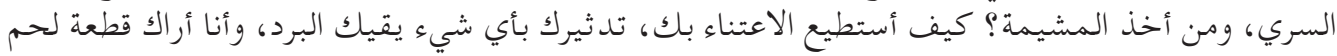

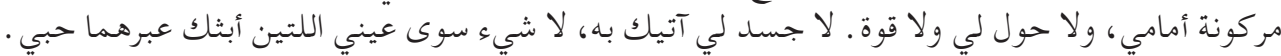

$$
\begin{aligned}
& \text { تبكين ! }
\end{aligned}
$$

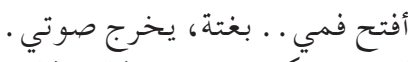

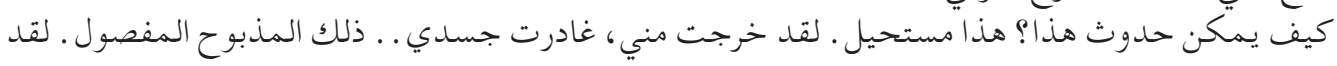

$$
\begin{aligned}
& \text { أصبحت غيري! } \\
& \text { كيف أشرح هذا؟ } \\
& \text { سأحاول . }
\end{aligned}
$$

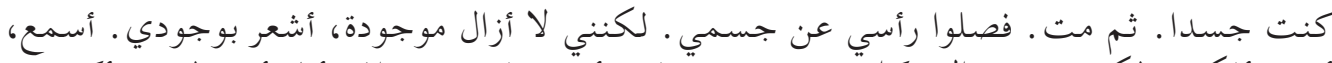

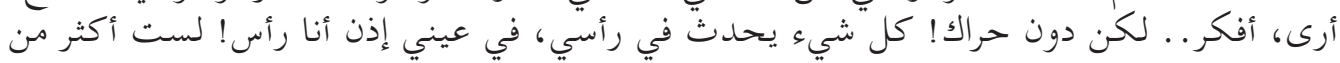

$$
\begin{aligned}
& \text { رأس! }
\end{aligned}
$$


«J'aurais voulu te voir naître entre mes bras, imprimer un baiser sur ton front et caresser ta peau douce. Comment le cordon ombilical a-t-il été coupé et qui a pris le placenta? Comment pourrais-je prendre soin de toi, t'envelopper dans quelque chose qui te protège du froid, alors que je te vois, telle un morceau de viande, recroquevillée devant moi qui suis sans défenses et sans forces, sans un corps pour te porter, sans rien à part mes yeux à travers lesquels je répands mon amour pour toi.

Tu pleures!

J'ouvre la bouche... Soudain ma voix se fait entendre.

Comment ceci a-t-il pu arriver? C'est impossible. Je suis sortie de moi-même, j'ai quitté mon corps...

Mon corps égorgé, détaché. Je suis devenue une autre !

Comment l'expliquer?

Je vais essayer.

J'étais un corps. Ensuite je suis morte. Ils ont détaché ma tête de mon corps. Mais je continue à être présente, je ressens ma présence. J'entends, je vois, je pense... Mais sans mouvement ! Tout se passe dans ma tête, dans mes yeux, donc je suis une tête! Rien de plus qu'une tête !»

Sulțāna prend directement la parole en s'adressant à sa fille. Le corps est l'élément qui crée la liaison entre mère et fille : le cordon ombilical et le placenta - symboles par excellence du lien matériel entre la mère et son enfant - sont explicitement nommés. La douleur de la séparation passe à travers les sensations corporelles transmettant le sentiment d'impuissance éprouvé par la mère, incapable de protéger son enfant. Les sens de la vue et de l'ouïe dominent le chapitre. Le sang de Sulțāna envahit tout le village, de même que le tintement des clochettes ayant poussé sur l'arbre à côté du cadavre (p. 21-22) :

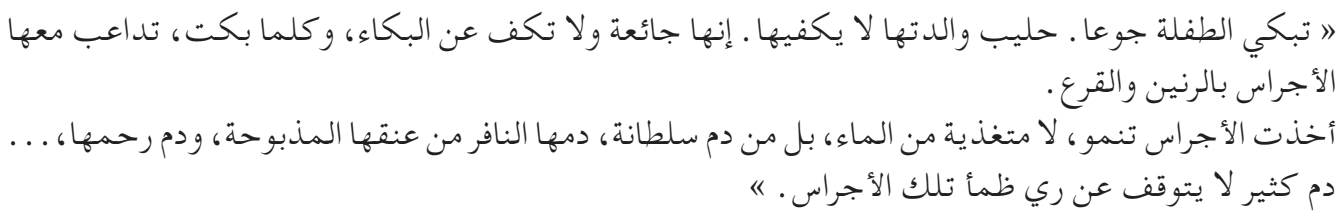

« L'enfant pleurait de faim. Le lait de sa mère ne lui suffisait pas. Elle était affamée et n'arrêtait pas de pleurer. Chaque fois qu'elle pleurait, les clochettes tintaient et vibraient avec elle.

Les clochettes commencèrent à pousser, nourries non pas de l'eau, mais du sang de Sulțāna, le sang qui jaillissait de son cou égorgé, le sang de son utérus... Beaucoup de sang qui ne cessait d'arroser la soif de ces clochettes. »

Le corps de Sulțāna ne cesse pas d'exister après sa mort, mais il se déverse sur le monde extérieur à travers son sang. Ici, l'écrivaine utilise l'image de l'arbre arrosé par le sang de la victime pour créer une scène qui se détache du langage du fantastique pour s'approcher de celui de l'horreur. De victime, la femme devient l'auteure de sa vengeance, en obligeant les habitants du village à subir les conséquences de l'injustice du crime d'honneur.

Dans les passages analysés jusqu'ici, le corps est représenté dans la dimension de la souffrance, expression de la douleur physique et psychologique de la victime et de son enfant. Le corps est décomposé en une série de parties, comme la tête, l'utérus, le cordon ombilical et le sang, qui forment une sorte de symbolique féminine de la souffrance. La différence du corps féminin est affirmée dans le but de re-signifier la symbolique 
historiquement marquée par le phallus. Par exemple, Luce Irigaray (1974) et Hélène Cixous (2010), qui se rattachent à la théorie de la différence ${ }^{12}$, affirment que le langage et l'écriture féminine sont l'expression du corps. Le texte reflète le corps, et la littérature devient un acte de réécriture et de relecture de la différence. Le corps est le contre-texte qui permet à la femme d'échapper au rôle, marqué par le silence et par le vide, qui lui a été attribué durant l'Histoire. Dans le roman, la protagoniste, après sa mort, refuse le silence qui lui a été imposé, en se déversant sur le monde à travers la couleur rouge. Cette couleur, en outre, est surchargée de symboles liés au corps féminin : rouge comme le sang de la vierge déflorée, sang des menstrues, sang de l'accouchement ; mais également couleur de l'amour et de la passion.

Dans le roman, on remarque que le corps est associé à la douleur quand il apparait dans une dimension collective, c'est-à-dire quand il est associé à la communauté. À l'intérieur du premier chapitre, le corps est le terrain de la lutte entre les composantes individuelle et collective. La femme qui a perdu sa virginité est punie à cause d'une coutume qui doit être respectée pour maintenir l'ordre social. En revanche, le corps jouit seulement quand il apparaît dans une dimension naturelle.

À ce propos, on note que la présence de l'élément naturel marque les stades principaux de la vie de Sultāna (p. 48-49). Au moment de sa naissance le jardin de la maison fleurit, en devenant un paradis (ğanna, p. 47) ; quand elle embrasse Ibrāhīm pour la première fois, le jasmin sous lequel ils sont abrités les enveloppe de ses branches (p. 59), tout comme il continue à grandir le jour des premières règles de Sultāna (p. 59). Mais la manifestation la plus remarquable advient lors de la première liaison sexuelle entre les deux amants, quand le parfum du jasmin envahit tout le village et réveille le désir des ses habitants (p. 60). Toutefois, le chapitre se termine en revenant sur le début du roman, c'est-à-dire à la scène de l'égorgement. Dès que la grossesse de Sulțāna est révélée aux yeux de la communauté, l'idylle naturelle finit et la tragédie collective commence (p. 62) :

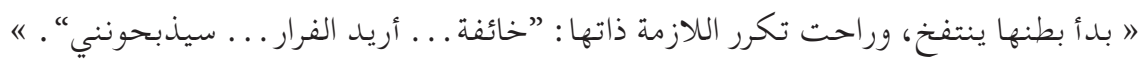

«Son ventre commença à gonfler et elle se mit à répéter le même refrain : “J’ai peur... Je veux m'enfuir... Ils vont m'égorger". »

Après la mort de Sulțāna et le début de la malédiction du rouge, les habitants du village sont effrayés par l'amour et le sexe. Le personnage de Samīra, l'une des jeunes femmes du village, représente cet état de «schizophrénie » vécu par les gens, partagés entre la peur de l'amour, source de déshonneur, et le désir (p.97):

12 Dans la vision des théoriciennes de la différence, comme Luce Irigaray, Hélène Cixous, Julia Kristeva, Luisa Muraro, la différence que la femme porte en elle et que la société patriarcale a toujours considérée comme un élément d'infériorité ne doit pas être dépassée, mais il faut lui attribuer un sens positif. Bien que la différence soit le lieu où pouvoir et violence se rencontrent et qu'elle puisse être à l'origine de l'oppression de l'autre, les théoriciennes de la différence la considèrent comme la clé pour la subversion et pour la naissance d'un nouveau sujet féminin. Elles introduisent donc l'idée d'un sujet incarné, avec des spécificités corporelles, qui se distingue de l'homme (voir BRAIDOTTI 2002, p. 92). 


$$
\begin{aligned}
& \text { " رفضت سميرة الزواج، مدافعة عن عذريتها ل لم تتقبل أن يلجها رجل، ويحصل على عذلريتها ـ الرجال وجدوا }
\end{aligned}
$$

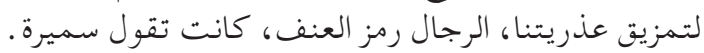

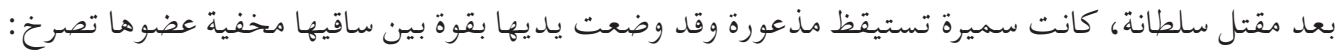

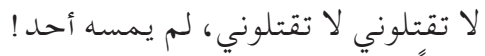

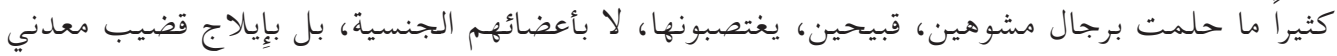

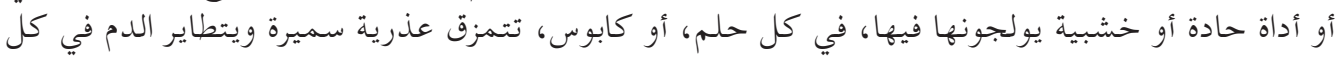

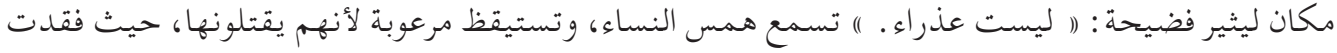

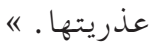

«Samīra avait refusé de se marier pour défendre sa virginité. Elle n'avait pas accepté qu'un homme la pénétrât et lui prît sa virginité. "Les hommes ont été créé pour déchirer notre virginité. Les hommes sont le symbole de la violence", disait Samīra.

Après le meurtre de Sulțāna, Samīra se réveillait effrayée, les mains posées avec force entre ses cuisses, cachant son organe et hurlant: "Ne me tuez pas, ne me tuez pas, personne ne l'a touché !"

Elle rêvait souvent d'hommes difformes et laids qui la violaient, non pas avec leurs organes sexuels, mais avec une barre de fer ou un outil pointu ou en bois avec lesquels ils la pénétraient. Dans chaque rêve, ou cauchemar, la virginité de Samīra se déchirait et le sang se répandait partout, pour provoquer le scandale : "Elle n'est pas vierge.". Elle entendait les autres femmes chuchoter et se réveillait remplie d'effroi car ils l'auraient tuée si elle avait perdu sa virginité. »

Dans ce passage, le corps est lu seulement à travers la logique communautaire: la femme est identifiée avec la virginité - le terme est répété cinq fois -, tandis que les hommes, regroupés dans un ensemble sans distinction, représentent le phallus qui vise à lacérer cette virginité. L'individu disparaît pour être réduit, par métonymie, à l'organe sexuel, qui est détaché de la dimension du plaisir. Du point de vue de la communauté, donc, le corps est cause d'angoisse en tant que source primaire du danger.

Pour retrouver, dans le roman, une dimension positive du corps, il faut attendre le début de la relation entre la fille de Sultāna et Hamza (p. 122) :

$$
\begin{aligned}
& \text { " سرب من الفراشات الميتة، الملونة، نبعت من قعر الشجرة ... [ . . }
\end{aligned}
$$

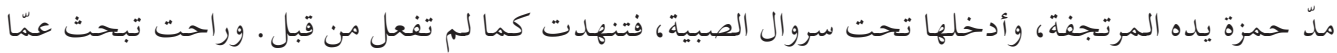

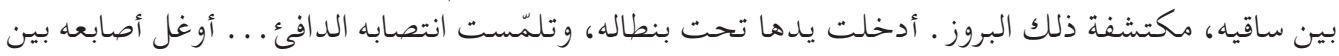

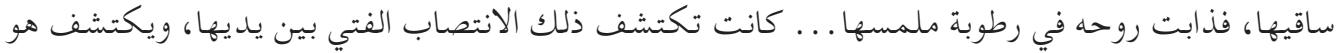

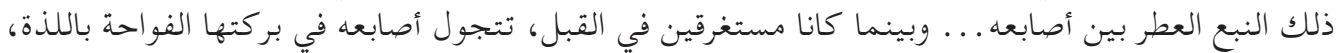

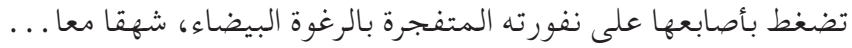

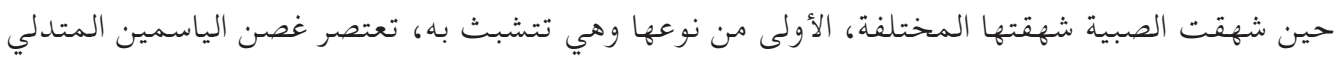

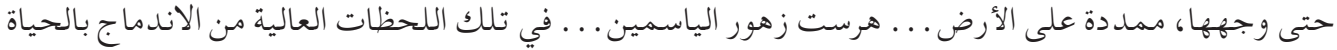

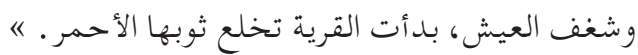

«Un vol de papillons morts, bariolés, jaillit du creux de l'arbre... [...]

Hamza tendit sa main tremblante et l'introduisit dans la culotte de la jeune fille qui haletait comme elle ne l'avait jamais fait auparavant. Elle commença à chercher ce qui était entre ses jambes à lui, découvrant cette protubérance. Elle introduisit sa main dans son pantalon, touchant sa tiède érection... Il enfonça profondément ses doigts entre ses jambes. Son âme se 
désagrégea au contact de l'humidité... Elle découvrait cette jeune érection entre ses mains, tandis qu'il découvrait cette source parfumée entre ses doigts... Pendant qu'ils étaient plongés dans les baisers, que ses doigts parcouraient cette bénédiction parfumée de plaisir et qu'elle serrait ses doigts autour de la fontaine de laquelle jaillissait de l'écume blanche, ils atteignirent l'orgasme ensemble.

Lorsque la jeune fille émit des soupirs différents, les premiers de ce genre, en s'accrochant à lui, elle pressa le rameau de jasmin qui surplombait son visage, alors qu'elle était étendue par terre... Elle écrasa les fleurs du jasmin... En cet instant sublime de fusion avec la vie et de désir de vivre, le village commença à se débarrasser de son vêtement rouge. »

Le langage du fantastique pénètre la narration, en générant un monde fictif dans lequel faire l'amour a un effet bénéfique sur la nature. Le corps est représenté dans sa dimension relationnelle, comme lieu de la découverte et de la connaissance de l'autre. Le rapport sexuel entre les deux jeunes gens est un parcours d'exploration réciproque, comme on le remarque à travers la répétition du verbe iktašafa (découvrir). L'organe sexuel féminin perd sa connotation négative de symbole de l'honneur en devenant une baraka (bénédiction). La nature, représentée par les papillons et le jasmin, est la seule dimension dans laquelle l'individu peut éprouver des sensations positives. Le contact sexuel avec l'autre devient la métaphore de l'affirmation du moi. C'est seulement grâce à l'amour et au plaisir que le village arrive à se libérer de la malédiction du rouge et à s'ouvrir à l'univers des couleurs. Même le personnage de la fille de Sulțāna, qui n'avait pas de prénom, acquiert son identité à travers la composante naturelle. Hamza découvre que le vagin de la fille dégage l'odeur du basilic (al-rayhạann) et décide de l'appeler Rayhạna. ${ }^{13}$

Toutefois, même si l'on trouve, dans le roman, des espaces pour l'affirmation du corps et, donc, de l'individu, l'auteure ne conclut pas son œuvre par une fin heureuse. L'instance individuelle n'est pas assez forte pour pouvoir briser les contraintes imposées à l'individu par la logique traditionnelle : l'histoire se répète donc, et Rayhāna subit le même destin que Sulțāna. Par contre, dans le dernier chapitre, c'est la logique du fantastique qui s'impose, en générant un univers parallèle dans les terres sauvages, dans lequel les femmes sont enfin libres et pratiquent l'amour sans entraves. Mais, comme le narrateur l'affirme, ce chapitre demeure relégué dans une dimension imaginaire car il ne s'est pas encore réalisé (p. 145). Dans la réalité, la femme n'a pas encore la possibilité de s'exprimer librement en tant qu'individu.

\section{Conclusion}

À l'intérieur du roman analysé, qui représente un exemple de la production romanesque syrienne récente, le corps fait l'objet d'une problématisation et joue un rôle central dans

13 Il faut remarquer que, dans le roman, les noms des personnages sont surchargés de signifiés allégoriques. On rencontre des noms liés à l'univers des contes de fées comme par exemple Tamīma, Mālika, Sulțāna ; des noms liés aux origines de la civilisation arabe et musulmane comme Ḥamza et Ibrāhīm ; et le nom de Rayhāna qui représente la dimension naturelle. 
l'œuvre. Il est envisagé en tant qu'emplacement ${ }^{14}$ de l'être humain, et sa crise représente la crise de l'individu, dans une société qui ne limite pas seulement la liberté de la femme, mais également celle de l'homme. À ce sujet on remarque que, bien que les écrivaines se focalisent sur la représentation du corps de la femme, elles pénètrent aussi dans l'intimité masculine.

Dans Banāt al-barārī, Mahā Hasan utilise le corps pour représenter le terrain de la lutte entre l'individu et la société. D'un côté, le corps est la source du plaisir ainsi que le moyen de la liaison avec l'autre, et, de l'autre côté, il est la cible du contrôle de la communauté. Mais ce n'est pas seulement la femme - en tant que symbole de l'honneur de la famille à travers sa virginité - qui subit la pression sociale. L'homme est également victime du code d'honneur qui lui impose un modèle précis de comportement, souvent lié à la violence. Le crime d'honneur symbolise donc l'échec de l'individu, qui perd son identité au contact de la collectivité.

Au niveau de la narration, cela se manifeste par l'impossibilité qu'ont les personnages d'instaurer des relations amoureuses et sexuelles dans les lieux de la vie communautaire : il n'y a pas d'exemple de liaisons passionnelles libres se déroulant dans le village, et qui soient légitimées par ses habitants. En revanche, la jouissance trouve sa place dans la nature. Pour affirmer sa propre personne ainsi que les droits de son corps, l'individu doit franchir la logique communautaire représentée, dans le roman, par les gens du village. La symbolique naturelle offre une deuxième alternative, une nouvelle dimension - fantastique dans ce cas - dans laquelle l'individu peut exister. La femme devient le symbole par excellence de la libération de la société parce qu'elle subit une double répression : celle du système patriarcal et celle de la communauté. À travers sa libération, qui ne peut se passer que dans un univers fantastique comme celui que l'auteure décrit dans le dernier chapitre (p. 145151), la femme prend aussi en charge la libération de l'homme.

Dans le domaine littéraire, la femme a déjà été choisie comme symbole de la libération sociale, même dans des œuvres signées par des auteurs hommes. Dans Ṭuqūs al-išārāt wa-ltahawwulāt, par exemple, Sa'd Allāh Wannūs présente la relation dialectique entre l'individu et les institutions sociales en s'appuyant sur la dimension sexuelle. L'auteur critique les institutions politiques et religieuses de sa société. L'un des personnages centraux est Almāsa, la femme du chef des Ašrāf (les descendants du Prophète), qui décide de devenir une prostituée pour enfin pouvoir vivre librement sa sexualité. Ce personnage féminin révolutionnaire représente la libération de l'individu de toute contrainte socio-religieuse et politique, à travers le corps. ${ }^{15}$

Le roman de Mahā Hasan s'inscrit donc dans la continuation d'un parcours littéraire en plein essor, dans lequel le corps - et notamment celui de la femme - est le terrain où se manifeste la dialectique entre l'élan individuel et la répression exercée par le contrôle

14 On se réfère ici à la théorie de l'emplacement d'Adrienne Rich (1994), selon laquelle le corps est le lieu principal d'énonciation du sujet où le matériel, le symbolique et le social se rencontrent.

15 Voir notamment le dialogue entre le moufti et Almāsa, une sorte de manifeste de la libération individuelle (S. WANNŪS 1994, p. 101-102). 
communautaire (patriarcal, religieux et politique). Par conséquent, la libération du désir est liée à la libération de l'individu social, tout comme la liberté individuelle est la condition de la liberté socio-politique.

\section{Bibliographie}

\section{Sources}

BARAKĀT Salīm, Fuqahāà al-z̧alām, Nicosie, Bīsān burs, 1985.

ǦARRĀH Amal, Al-riwāya al-mal ūna, Beyrouth, Dār al-sāqī, 2010.

ḤASAN Mahā, Banāt al-barārī [Les filles des prairies], Beyrouth, Riyāḍ al-Rayyis, 2011.

WANNous Dīma, Kursī, Beyrouth, Dār al-ādāb, 2009.

WANNous Sa'd Allāh, Ṭuqūs al-išārāt wa-l-tahawwulāt, Beyrouth, Dār al-ādāb, 1994.

YĀSīn ḤASAN Rūzā, Hurrās al-hawā', Beyrouth, Riyāḍ al-Rayyis, 2009.

YAZBIK Samar, Rā’h hat al-qurfa, Beyrouth, Dār al-ādāb, 2008.

BīṬ̂AR Hayfā', Imra'a min hāẹā al-'aṣr, Beyrouth, Dār al-sāqī, 2007.

\section{Études}

BARŪT Ǧamal, 2001, "Al-riwāya al-sūriyya wa-l-tārīh : al-as'ila al-ūlā », in CHEHAYED, Jamal et TOËLLE, Heidi, Al-riwāya al-suriyya al-mu'āsira. Al-ğud̄ūr al-țaqāfiyya wa-l-taqaniyyāt al-riwā̄iyya al-ğadīda [Le roman syrien contemporain. Racines culturelles et rénovation des techniques narratives], Damas, Institut français d'études arabes, p. 75-82.

BitAR OTTOSson, Astrid, 2005, I Can Do Nothing against the Wish of the Pen. Studies in the Short Stories of Widād Sakākīnī, Uppsala, Uppsala Universitet (coll. Studia Semitica Upsaliensia 21).

BRAIDOTTI Rosi, 2002, Nuovi soggetti nomadi. Transizioni e identità postnazionaliste, Bologna, Luca Sossella Editore.

CALVINo Italo, 2002 [1 $1^{\text {re }}$ éd. 1988], Sulla fiaba, Milano, Mondadori.

CENSI Martina, 2013, Les représentations du corps dans le roman des écrivaines syriennes contemporaines, Thèse de doctorat de troisième cycle, Paris, Institut national des langues et civilisations orientales, et Venise, Université Ca' Foscari.

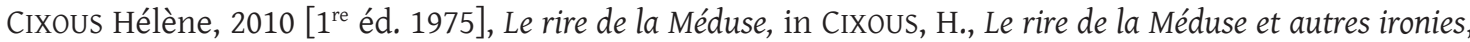
Paris, Galilée, p. 35-68.

DARRĀĞ Fayșal, 2001, " "Qașr al-matar", al-tārīh al-mahzūm fì al-mustabidd al-muntașir » [Qașr al-mațar ou l'histoire vaincue par le despote victorieux], in CHEHAYED, Jamal et TOËLLE, Heidi, Al-riwāya al-suriyya al-mu'āșira. Al-ğud̄ūr al-țaqāfiyya wa-l-taqaniyyāt al-riwāìyya al-ğadīda [Le roman syrien contemporain. Racines culturelles et rénovation des techniques narratives], Damas, Institut français d'études arabes, p. 45-74.

HALLAQ Boutros, 2001, «Al-kitāba: al-wāî̀ wa-țaqāfat al-ğasad » ["L'écriture : la conscience et la mémoire du corps], in CHEHAYED, Jamal et TOËLLE, Heidi, Al-riwāya al-suriyya al-mu āșira. Al-ğud̄ūr al-țaqāfiyya wa-l-taqaniyyāt al-riwāilyya al-ğadìda [Le roman syrien contemporain. Racines culturelles et rénovation des techniques narratives], Damas, Institut français d'études arabes, p. 179-192. 
IRIGARAY Luce, 1974, Speculum. De l'autre femme, Paris, Éditions de Minuit.

Jolles André, 2003 [éd. originale 1930], Forme semplici, in JolLES, André, I travestimenti della letteratura. Saggi critici e teorici (1897-1932), Milano, Mondadori.

RICH Adrienne, 1994, Notes toward a Politics of Location in RICH, Adrienne, Blood, Bread and Poetry: Selected Prose (1979-1985), New York, W. W. Norton, p. 210-231.

SCOTT Joan W., 1986, « Gender: A Useful Category of Historical Analysis », American Historical Review 5/91, p. 1053-75.

TODOROV Tzvetan, 1970, Introduction à la littérature fantastique, Paris, Seuil, 1970.

ZEMMOUR Zine-Eddine, 2002, « Jeune fille, famille et virginité. Approche anthropologique de la tradition », Confluences Méditerranée 41, p. 65-76.

ZEYDAN, Joseph, 1995, Arab Women Novelists : The Formative Years and Beyond, Albany, State University of New York Press. 